\title{
Pharmacovigilance as a tool for safety and monitoring: a review of general issues and the specific challenges with end-stage renal failure patients
}

\author{
This article was published in the following Dove Press journal: \\ Drug, Healthcare and Patient Safety \\ 12 April 2013 \\ Number of times this article has been viewed
}

\author{
Dalia Jacob' \\ Belén Marrón ${ }^{2}$ \\ Jay Ehrlich' \\ Peter A Rutherford ${ }^{3}$ \\ 'Baxter Healthcare Corporation, \\ Deerfield, IL, USA; ${ }^{2}$ Baxter Healthcare \\ Corporation, Madrid, Spain; ${ }^{3}$ Baxter \\ Healthcare SA, Zurich, Switzerland
}

Correspondence: Peter Rutherford Medical Director (Renal) - Europe, Middle East and Africa, Baxter Healthcare SA, Thurgauerstrasse 130, Zurich 8010 , Switzerland

Tel +4I 448786395

Fax +4I 448786379

Email peter_rutherford@baxter.com

\begin{abstract}
Pharmacovigilance is instrumental in helping to ensure patient safety for both newly released drugs and those that are well established in the market. However, while pharmacovigilance procedures are strictly regulated in the clinical trial setting, post-marketing adverse event reporting is not well implemented or enforced. As such, the underreporting of adverse events, in relation to drugs that are on the market, is estimated to be in the region of $90 \%$. The identification of drug safety issues in patients with complex diseases and extensive comorbidities is therefore particularly challenging. Dialysis patients - those with end-stage renal disease and often other comorbidities such as diabetes, hypertension, and cardiovascular disease - are a population with significant treatment challenges. Patients receive dialysis using complex medical devices (eg, a peritoneal dialysis home cycler) and also receive a range of pharmaceutical agents as part of dialysis itself (eg, peritoneal dialysis solutions). Many of the pharmaceutical agents used to treat these patients have been developed in populations without these complications and, therefore, an extensive knowledge of potential problems and contraindications in the dialysis population is lacking. It is important that the nephrology community understands the concept of pharmacovigilance - the pharmacologic science relating to the detection, assessment, understanding, and prevention of adverse effects, particularly long-term and short-term side effects, of medicines. Health care professionals (HCPs) and providers, pharmaceutical companies, global regulatory agencies, and the patients themselves all play unique and critical roles in this process. This review defines the science of pharmacovigilance and the process of adverse event reporting, highlights the new directions that pharmacovigilance has taken, and provides insight for HCPs managing dialysis patients into the important role that they play in helping to shape the understanding of a drug's safety profile in order to continually enhance patient safety.
\end{abstract}

Keywords: adverse events, drug safety, peritoneal fluids, comorbidities, dialysis, end-stage renal disease

\section{Introduction}

Pharmacovigilance is defined as the pharmacological science relating to the detection, assessment, understanding, and prevention of adverse effects, particularly long-term and short-term side effects, of medicines. ${ }^{1}$ While not well understood by those outside of the drug safety world, pharmacovigilance plays a pivotal role in helping to ensure patient safety for both newly released drugs and those that are well-established in the market. Pharmacovigilance involves consumers, health care professionals (HCPs), pharmaceutical companies, and global regulatory agencies, each of whom plays a unique and critical role in this process. This review defines the science of 
pharmacovigilance and the process of adverse event reporting, with particular reference to the challenges of reporting complex diseases such as end-stage renal disease (ESRD). This review highlights the new directions that pharmacovigilance has undertaken, and provides insight for HCPs into the important role that they play in helping to shape the understanding of a drug's safety profile in order to continually enhance patient safety.

Patients with chronic kidney disease (CKD) and ESRD are a population with significant challenges and complex therapeutic regimens. Table 1 summarizes some of the specific challenges relating to the identification of drug safety issues in this group of patients. The presence of multiple comorbidities such as cardiovascular disease, hypertension, and diabetes requires the use of a variety of pharmaceutical interventions in addition to those needed to manage renal insufficiency itself. Adding to this complex situation is the impact of chronic renal replacement therapy, which has its own set of adverse effects, but where drug effects may play either a causative role or may interact with an evolving complication. Many of the drugs used to treat patients with CKD/ESRD have been developed in populations with normal renal function and/or without significant comorbidities, and also without the additional challenge of understanding the altered pharmacokinetic effects due to impaired renal excretion and changes in volume of distribution and fluid balance. The impact of a drug safety issue in a patient with end-stage

Table I Factors relating to drug safety issues in end-stage renal failure patients

\begin{tabular}{|c|c|}
\hline Related factor & Potential issue \\
\hline $\begin{array}{l}\text { Demographics of end-stage } \\
\text { renal failure }\end{array}$ & $\begin{array}{l}\text { Progressively elderly dialysis } \\
\text { incident population with frequent } \\
\text { comorbidities }\end{array}$ \\
\hline Comorbid illness & $\begin{array}{l}\text { Polypharmacy is common in } \\
\text { patients with renal disease and } \\
\text { such patients are less adaptable to } \\
\text { pathophysiological changes }\end{array}$ \\
\hline $\begin{array}{l}\text { Clinical evidence base for } \\
\text { side effects }\end{array}$ & $\begin{array}{l}\text { Often based on studies of patients } \\
\text { with normal kidney function or } \\
\text { less severe renal impairment }\end{array}$ \\
\hline $\begin{array}{l}\text { Complications of chronic } \\
\text { kidney disease }\end{array}$ & $\begin{array}{l}\text { Hypertension, anemia, and } \\
\text { metabolic bone disease require } \\
\text { specific interventions with } \\
\text { frequent drug and dose alterations }\end{array}$ \\
\hline Impact of dialysis therapy & $\begin{array}{l}\text { Complex medical devices are used } \\
\text { and specific pharmaceutical agents } \\
\text { are part of the dialysis procedure }\end{array}$ \\
\hline $\begin{array}{l}\text { Causation of, or interaction } \\
\text { with, potential long-term } \\
\text { complications of dialysis therapy }\end{array}$ & $\begin{array}{l}\text { For example, myocardial stunning } \\
\text { with short hours hemodialysis and } \\
\text { encapsulating peritoneal sclerosis } \\
\text { with long term peritoneal dialysis }\end{array}$ \\
\hline
\end{tabular}

renal failure may operate through different mechanisms and could include:

1. Direct patient harm from the clinical sequelae of the adverse event

2. An interaction with the dialysis therapy itself (for example, the interaction of angiotensin-converting-enzyme [ACE] inhibitors with specific polyacrylonitrile hemodialysis membranes, causing an acute hypersensitivity reaction)

3. An acceleration of the loss of residual kidney function this may be measured by standard current biochemical methods or in the future with novel sensitive biomarkers of renal damage. ${ }^{2}$

As information related to the use of medications in the $\mathrm{CKD} /$ dialysis population is at best limited, it is important that nephrologists and other HCPs managing patients with end-stage renal failure understand the concept and implementation of pharmacovigilance. This will contribute to enhanced patient safety for this high risk population, and mirrors the drive by professional nephrology organizations to develop patient safety indicators to improve CKD-specific patient safety. ${ }^{3}$ Drug adverse events are critical elements of the draft CKD patient safety indicators developed by the American Society of Nephrology. This further emphasizes the need for greater understanding and implementation of pharmacovigilance in everyday clinical practice.

\section{Pharmacovigilance: then and now}

Regulatory agencies, such as the US Food and Drug Administration (FDA) and the European Medicines Agency (EMA), have many responsibilities, including the protection and promotion of public health through the evaluation and supervision of medicines for human use. ${ }^{4}$

The roles of such regulatory agencies have evolved over the last century to focus on consumer protection and patient safety. In the early 1900s, the FDA (Bureau of Chemistry) focused regulatory enforcement on foods that were thought to pose a greater public health problem than adulterated or misbranded drugs. However, in 1938 the Food, Drug and Cosmetic Act brought cosmetics and medical devices under control, and required that drugs be labeled with adequate directions for safe use. ${ }^{5}$ Amendments to this law have allowed the FDA to continue to refine its ability to protect public health through regulating medicines for human use.

The EMA was founded in 1995; it is a decentralized body of the European Union (EU). Its main responsibility is to oversee the protection and promotion of public and animal health through the evaluation and supervision of 
medicines for human and veterinary use. The EMA can be considered the hub of a European medicines network comprising over 40 national competent authorities, the European Commission, the European Parliament, and a number of other decentralized EU agencies. The Agency works closely with its European partners to build the best possible regulatory system for medicines for Europe and to protect the health of its citizens. ${ }^{4}$

Pharmacovigilance has evolved from a largely recordkeeping function - where the purpose of pharmacovigilance was mainly to ensure the processing and submission of individual case safety reports and aggregate reports - to the present, where pharmacovigilance now focuses on proactively identifying safety issues ("signals") and taking actions to minimize or mitigate risk to patients. This evolution has been especially rapid since the early 2000 s.

\section{Pharmacovigilance in the post-marketing setting}

Pharmacovigilance in the post-marketing setting (ie, once a drug has been approved and is available in the market place) starts with the receipt of adverse event information (a "case" or individual case safety report) from many different sources including consumers, HCPs, regulators, literature, and media such as the Internet. Since reporting of adverse events in the post-marketing setting is mainly a voluntary process, analysis of these data is limited by the willingness of an HCP (or any other reporter) to actually report the case and also to provide enough detail to allow for assessment of whether or not a drug may actually have caused or contributed to an adverse event.

An adverse event is defined as any untoward medical occurrence in a patient who has been administered a medicinal product and does not necessarily have to have a causal relationship with this treatment. An adverse event can therefore be any unfavorable and unintended sign, symptom, disease, or outcome of death temporally associated (ie, associated in terms of timing) with the use of a medicinal product, whether or not considered to be caused by this medicinal product. ${ }^{6}$ The important distinction between an adverse event and an adverse reaction is that a reaction indicates a causal association between the drug and the reported event.

During the development of a new drug, clinical trials are performed to evaluate drug safety and efficacy. It is mandatory that all adverse events that occur during clinical trials are reported, allowing the company and regulators to have a more complete picture of the adverse event profile, as well as to determine the frequency (ie, "incidence rate") in the study population. These factors allow an educated assessment of the risks and safety profile of the drug when compared to the demonstrated benefits.

There are, however, limitations associated with defining a drug's side effect profile based solely on clinical trial data. Clinical trials typically include a small number of subjects relative to the population that will be prescribed the drug in the post-marketing setting, and while commonly occurring adverse events may be identified in clinical trials, rare adverse events may not be manifested until the drug is used by a much larger population or for a longer time period. In addition, clinical trial subjects may not exhibit the extensive comorbidities that the general population may have. This is extremely relevant for patients with CKD stages 3-5 and even more so for those on dialysis - these patients are invariably excluded from trials, their duration of therapy may be significantly longer than the maximum follow-up time period present in the relevant clinical trials, and the drug they are being prescribed may not have been studied in a CKD/ dialysis population. Thus, post-marketing pharmacovigilance is particularly relevant to identify potential safety issues in dialysis patients, and to highlight the need for increased patient monitoring and specific warnings.

While post-marketing pharmacovigilance can be extremely valuable in identifying a safety signal, it is important to understand its limitations. Reports in the popular press about a particular drug being recalled or causing a particular adverse reaction may cause an influx in adverse event reports as HCPs "think back" about patients that they have seen, leading to potential false safety signals. In addition, the true frequency of an adverse event cannot be determined from post-marketing data, as neither the numerator (total number of adverse events that actually occurred) nor the denominator (total number of doses actually administered or total number of patients who took the drug) are unavailable. Many countries have registries of dialysis patients, which examine dialysis practice and dialysis-related clinical outcomes to allow observations of trends over time and comparison both within and between dialysis units. However, although they contain substantial data, they cannot be used to examine specific drug-related adverse events as they are not recorded as such in the registry database. Furthermore, precise data on drug use is gathered in only a minority of such registries.

Thus, post-marketing data are essential to allow for continuous evaluation of the safety of marketed products. They can help confirm or put into perspective safety issues or signals discovered during clinical trials, identify safety signals not previously recognized, or identify "new" signals 
within specific patient populations that had not been studied during clinical trials. A safety signal may manifest in many different ways: as a new, previously unknown adverse event, a known adverse event that is now being reported at a higher frequency, a new drug interaction, and/or a new adverse event identified within a specific dialysis sub-population or associated with a specific dialysis modality.

\section{Pharmacovigilance reporting}

An important distinction between post-marketing and clinical trial adverse event reporting is that reporting is mandatory in a clinical trial setting, but largely voluntary in the post-marketing setting. As such, underreporting of postmarketing adverse events is a well-recognized phenomenon: while difficult to estimate, the level of underreporting in the spontaneous reporting systems is likely in excess of $90 \%{ }^{7}$ Despite this massive underreporting, an analysis of 21 drugs withdrawn in France between 1998 and 2004 concluded that 19 withdrawals were linked to post-marketing adverse event case reports. $^{8}$

One major reason for underreporting is that many reporters believe there is no need to report an adverse event if the side effect is known and well-established. Unfortunately, this underreporting does not allow for the identification of a change in the frequency of an established adverse event in either the population in which a drug was studied (eg, due to a change in medical practice, introduction of other medications in the treated population, or a drug interaction), nor does it allow an appreciation of that adverse event in new or unintended patient populations. Adverse events should, therefore, always be reported if there is a suspicion that the drug caused or contributed to the event. Without such complete reporting, an accurate frequency of occurrence of a known adverse event cannot be fully understood.

There is an obvious need to continue to raise awareness of and promote adverse event reporting in order for a pharmaceutical company to fully investigate and understand a potential safety issue. Given the low percentage of postmarketing adverse events reported, consideration should be given to implementing HCP training and education to emphasize that adverse event reporting plays an important and impactful role in drug development and patient safety.

\section{Role of the HCP}

Many HCPs do not fully appreciate the important role they play in helping define the benefit-risk profile of a drug. Unlike clinical trials, where both a company and clinical trial regulations can set expectations regarding the medical details being reported, in post-marketing cases, even when an adverse event is reported, the details provided are solely at the discretion of the reporter. This may result in a lack of detail, which can lead to difficulty in assessing whether or not a causal relationship exists between the administration of the drug and the onset of the adverse event.

There are regulations in place that require companies to follow up with the reporter of an adverse event in order to obtain enough information to help determine the causal assessment; however, regulators require "minimum" information to process and report a case, including identifying the drug that was considered suspect, the patient (using an identifier such as "an elderly male patient" or "a Caucasian female patient" but not needing to identify the patient by name), a drug product, a reporter, and the adverse event itself.

Worldwide, a variety of regulations exist that companies must abide by; however, the International Conference on Harmonization ( $\mathrm{ICH}$ ) does set out guidelines that many authorities have adopted directly or incorporated into their regulations. ${ }^{6}$ For example, all authorities have adopted the ICH definition for what should be considered a serious adverse event. It is important to note that this definition is not necessarily the same as what a clinician may feel is "serious" in terms of the impact to the patient. The generally agreed criteria for seriousness appear in ICH guideline E2A. ${ }^{6}$ A serious adverse event (experience) or reaction is any untoward medical occurrence that:

- results in death;

- is life-threatening;

- requires inpatient hospitalization or results in prolongation of existing hospitalization;

- results in persistent or significant disability/incapacity;

- is a congenital anomaly/birth defect;

- is a medically important event or reaction.

These types of definitions are extremely important within the regulatory and pharmaceutical industry since many of these types of designations dictate whether an adverse event report should be submitted to an authority on an expedited basis (which under most circumstances would mean a report should be submitted within 15 days of receiving an adverse event) or submitted in a periodic manner (as part of an aggregate report that contains a collection of adverse event cases that may be submitted on a quarterly to annual basis).

The decision to report an adverse event can be a difficult one for a clinician, especially when many of the events seen are considered to be typical or "expected.” While many nephrologists view events such as peritonitis, fever during hemodialysis, breathlessness, abdominal pain, or edema as a "normal" 
or even frequent occurrence in a dialysis patient, these are still adverse events - untoward medical occurrences - and should be reported. The role, and indeed responsibility, of the clinician in reporting adverse events in dialysis patients should be included in undergraduate and postgraduate nephrology education. In addition, professional nephrology societies could encourage reporting of adverse events through their regular postgraduate meetings and publications.

\section{Role of patients}

It is also important to consider the role of dialysis patients in the identification of adverse events and potential drug safety issues. Dialysis patients are well-informed about their condition and its management and should be educated to report changes in their condition to their dialysis unit. A precise history from the patient of a clear temporal relationship between symptoms and drug exposure can be particularly helpful in determining causation. In addition, patient-reported outcomes (ie, those related to self-perception and without clinical interpretation) are another potential data source for detecting adverse events. There is some interest in this area and in particular the potential for direct patient reporting of outcomes for pharmacovigilance purposes. ${ }^{9}$ Direct patient reporting does occur in the US and, since the introduction of new EU pharmacovigilance legislation, to some extent in European countries. However, there are legal, regulatory, and practical concerns which need to be addressed.

\section{Pharmacovigilance and signal detection}

A safety signal is defined as safety information suggesting a new potential causal association between a medical event and a medicinal product that may represent a change in the established safety profile of that product. A confirmed safety signal is safety information that has been analyzed and is reasonably considered to demonstrate a causal association between a medicinal product and a medical event or concept. ${ }^{10}$

There are many different approaches used in signal detection, including data mining tools and manual signal detection methods. The basis of signal detection, however, should focus on examining adverse events from both a qualitative and quantitative standpoint. It does not take a pharmacovigilance expert to recognize a potential issue if the baseline number of cases received per month associated with a particular drug and adverse event is 10 for many months, and then the following month 60 such cases are received. The challenge is to analyze these cases in conjunction with any changes in the reporting environment. For example, if there was recent training at a hospital on reporting adverse events, it would not be surprising to see an increase in the number of cases reported from that hospital; that is reporting bias. Reporting bias, however, should not negate the quantitative safety signal identified. The next step is to analyze the cases and ask questions such as:

- Are there any factors that are similar in these cases?

- Did the patients use drugs with the same lot number?

- Is there a problem with, or change in, the technique of drug administration?

- Did the adverse event abate when the drug was discontinued, and/or did it return or worsen when the drug was administered again?

These types of questions help to determine whether or not a causal relationship exists between the drug and the adverse event. With this degree of information, full analysis can begin as to whether the drug is causing a particular event, and what actions should be taken. Once a signal is confirmed to reflect a safety issue, systems must be in place to ensure rapid communication with stakeholders. The European Commission, in close consultation with the EMA, EU Member States, and interested parties, developed a guideline on good pharmacovigilance practices, Module IX - Signal management. ${ }^{11}$ This guideline dictates that:

\begin{abstract}
Marketing authorization holders should communicate signals, that may have implications for public health and the benefit-risk profile of a product, immediately to the competent authorities as an Emerging Safety Issue, and when appropriate this should include proposals for action. The outcomes of signal assessment involving new or changed risks and risks that have an impact on the benefitrisk balance of the concerned active substance/medicinal products should be communicated to the public, including HCPs and patients, as well as to the concerned marketing authorization holders. ${ }^{11}$
\end{abstract}

An example of this process in action was the withdrawal of a specific lot of the peritoneal dialysis (PD) solution, Nutrineal $^{\text {TM }}$ (Baxter Healthcare Corporation, Deerfield, IL, USA) which occurred in Europe in October 2010. ${ }^{12}$ Baxter began receiving a number of reports of aseptic peritonitis associated with one specific lot of Nutrineal. While the reporters informed Baxter of the peritonitis, many gave extremely limited details, providing no information on laboratory investigations, minimal information on whether the patient improved upon discontinuation of the Nutrineal, not providing information on other PD solutions that may have played a role in the adverse event, and not confirming the specific lot of Nutrineal in use at the time of the events. The lack of such 
details made it extremely challenging to analyze this signal; however, as further details were collected during follow-up calls to HCPs, Baxter was able to identify the lot association and take necessary action to withdraw this lot from the market. This real-life example highlights the benefits of identifying adverse events and also the need for HCPs to report not only the adverse event, but additional details, such as lot numbers, temporal relationship between drug administration and the event, and actions taken to mitigate the adverse event (eg, medications used to treat the symptoms and discontinuation of the suspect drug) in order to help in the identification of a signal. Geerse et $\mathrm{al}^{12}$ pointed out another important link: that PD units should use their own clinical practice data to continuously monitor trends in peritonitis (rates, prevention strategies, and outcomes) and to combine and analyze them across units. In this specific example, the first unit to report cases had noticed such a change and an unusually high number of culture-negative cases. While all individual adverse events should be reported, if a change in rate or clinical characteristics of a known PD complication (eg, peritonitis) occurs it should be noted in the report to the manufacturer.

It is also important to highlight that while signals can occur in a rapid manner, there are many evaluations that occur on multiple levels to ensure that the benefit-risk balance of a drug remains positive. Adverse events are not only evaluated on a case-by-case basis, but on an aggregate level as well. Many companies have different data mining techniques to assist evaluation on an ongoing basis, and regulatory authorities themselves are continuously evaluating data to ensure that no new signals emerge from the adverse events they receive.

\section{Examples of adverse events from post-marketing data leading to action}

As post-marketing pharmacovigilance has continued to evolve, the public has become more aware of adverse events and drug safety. The popular press has highlighted instances in which safety signals identified using post-market safety data have identified safety concerns that had not been identified in clinical trials, leading to changes in or sometimes withdrawal of a product from the market. Within renal practice, there are many examples where post-marketing safety data have led to changes or drug withdrawals.

\section{Icodextrin and device interaction}

Extraneal $^{\mathrm{TM}}$ (icodextrin) (Baxter Healthcare Corporation) is a PD solution containing the colloid osmotic agent icodextrin, a starch-derived water-soluble glucose polymer. While Extraneal was closely monitored and assessed during clinical trials, an unanticipated, rare adverse event did not emerge until Extraneal was used by a larger population. Icodextrin, the osmotic agent in Extraneal, is metabolized into oligosaccharides including maltose and other higher molecular weight molecules. Certain glucometers are considered "nonspecific" and measure not only glucose but also metabolites such as maltose. The presence of maltose can lead to falsely elevated glucose readings, ${ }^{13}$ which could in turn lead to the administration of more insulin than needed in patients using these glucometers. Administration of more insulin than needed can cause hypoglycemia which may lead to loss of consciousness, coma, neurologic damage, and death. Additionally, falsely elevated blood glucose measurements due to maltose interference may mask true hypoglycemia, which left untreated can result in similar consequences. Falsely elevated glucose levels may be measured up to 2 weeks following cessation of icodextrin therapy.

This particular issue did not occur in the Extraneal clinical trials, but was identified as Baxter began receiving cases of falsely elevated glucose readings after product launch. This life-threatening issue is best exemplified by a published case report of a 59-year-old patient on Extraneal who was admitted for an elective procedure. ${ }^{14}$ During the preoperative period she communicated to the HCPs that, due to her Extraneal use, she required the use of a specific glucometer. Unfortunately, when the patient was transferred postoperatively to the intensive care unit (ICU) this message was not transferred with her. The readings on the nonspecific glucose handheld monitors in the ICU provided an overestimation of the glucose level, leading to the administration of too much insulin. The patient developed hypoglycemic encephalopathy and eventually died at an extended care nursing facility. ${ }^{14}$

This safety signal led to the implementation of many actions intended to enhance patient safety, including updating drug labeling with warnings. Additionally, Baxter has provided educational material to all patients and nephrologists whose clinics use Extraneal to help mitigate this risk through continued education on this otherwise unrecognized issue, and continues to work with different regulators to educate on the use of these nonspecific glucose monitors.

\section{Quinolones and tendon rupture}

The use of quinolone antibiotics has been associated with the development of tendon rupture. This risk was identified largely based on post-marketing rather than clinical trial 
data and illustrates an additional important point - many clinicians may not consider reporting a tendon rupture to the manufacturer of an antibiotic, believing that it is "biologically implausible," yet this adverse event was first reported in medical journals as case reports seen in the post-marketing setting. This adverse event has led to boxed warnings in all quinolone labels. This is of particular relevance in dialysis patients since quinolone use is not uncommon in this population. ${ }^{15}$

\section{Conclusion}

Pharmacovigilance benefits all key stakeholders within the health care arena, including patients, the HCPs taking care of these patients, the worldwide regulatory agencies who approve drugs, and the pharmaceutical companies that develop and market the drugs. Over the last 10 to 15 years, pharmacovigilance activities have evolved significantly from focusing on simply the reporting of individual case reports within a prescribed timeframe to a more proactive environment, including signal detection and risk management activities. While clinical trials allow a company to understand the initial safety profile of a drug, it is post-marketing data that truly help frame the full benefit-risk profile of a drug in the real world setting. Dialysis patients and those with CKD present unique challenges in management and care, so nephrologists need to be constantly vigilant and report adverse events rapidly, accurately, and thoroughly. The dialysis setting can be a high-risk clinical environment and there is increasing focus by HCPs, hospitals, and statutory bodies on implementing patient safety improvement initiatives. A recent review ${ }^{16}$ commented that dialysis patients take between six and ten different drugs daily and observed the high rate of potential drug safety issues. The authors recommended specific actions within dialysis units to improve patient safety, including the need for timely and accurate reporting and investigation of critical and adverse events.

While HCPs undergo training for their specialty of choice and are trained on many different and important aspects of medicine, it is increasingly obvious that one area requiring more attention is the need to report adverse events associated with drug administration. The ever-present underreporting of adverse events highlights the need to continue to promote the specialty of pharmacovigilance and to help HCPs understand the role that pharmacovigilance plays to help ensure patient safety. Pharmacovigilance signal detection activities incorporate aspects of both quantitative and qualitative analyses to interpret whether a drug may be causing an adverse event, and how that may alter the benefit-risk profile of the drug. Together, the HCPs committed to adverse event reporting, the regulators, and the pharmaceutical company can help shape the safety of drugs being used by patients.

\section{Acknowledgments}

The authors would like to thank Cathy Hoff of Baxter Healthcare Corporation for medical writing and editorial support. Further editorial assistance was provided by Angela Rogers of Gardiner-Caldwell Communications, United Kingdom and was funded by Baxter Healthcare Corporation.

\section{Disclosure}

Dalia Jacob, Jay Ehrlich, and Peter Rutherford are employees of Baxter Healthcare Corporation, and hold stock and/or stock options in Baxter Healthcare Corporation. Belén Marrón is a former employee of Baxter Healthcare Corporation. The authors report no other conflicts of interest in this work.

\section{References}

1. World Health Organization. The Importance of Pharmacovigilance Safety Monitoring of Medicinal Products. Geneva: World Health Organization; 2002. Available from: http://apps.who.int/medicinedocs/ pdf/s4893e/s4893e.pdf. Accessed March 6, 2013.

2. Tesch GH. Review: Serum and urine biomarkers of kidney disease: a pathophysiological perspective. Nephrology (Carlton). 2010;15(6): 609-616.

3. Fink JC, Joy MS, St Peter WL, Wahba IM; ASN Chronic Kidney Disease Advisory Group. Finding a common language for patient safety in CKD. Clin J Am Soc Nephrol. 2012;7(4):689-695.

4. European Medicines Agency [webpage on the Internet]. London: European Medicines Agency; 2013. Available from: http://www.ema.europa.eu/ ema/index.jsp?curl=pages/about_us/general/general_content_000091. jsp\&murl=menus/about_us/about_us.jsp\&mid=WC0b01ac0580028a42. Accessed December 6, 2012.

5. US Food and Drug Administration [webpage on the Internet]. Silver Spring: US Food and Drug Administration; 2013. Available at: http:// www.fda.gov/AboutFDA/WhatWeDo/History/default.htm. Accessed December 6, 2012.

6. International Conference on Harmonisation of Technical Requirements for Registration of Pharmaceuticals for Human Use. Clinical Data Safety Management: Definitions and Standards for Expedited Reporting E2A. Geneva: International Conference on Harmonisation of Technical Requirements for Registration of Pharmaceuticals for Human Use; 1994. Available from: http://www.ich.org/fileadmin/Public_Web_Site/ ICH_Products/Guidelines/Efficacy/E2A/Step4/E2A_Guideline.pdf. Accessed March 6, 2013.

7. Hazell L, Shakir SA. Under-reporting of adverse drug reactions: a systemic review. Drug Saf. 2006;29(5):385-396.

8. Olivier P, Monastruc JL. The nature of the scientific evidence leading to drug withdrawals for pharmacovigilance reasons in France. Pharmacoepidemiol Drug Saf. 2006;15(11):808-812.

9. Banerjee AK, Ingate S. Web-based patient-reported outcomes in drug safety and risk management: challenges and opportunities? Drug Saf. 2012;35(6):437-446.

10. Council for International Organizations of Medical Sciences. Practical Aspects of Signal Detection in Pharmacovigilance. Geneva: Council for International Organizations of Medical Sciences; 2010. 
11. European Medicines Agency and Heads of Medicines Agencies. Guideline on Good Pharmacovigilance Practices (GVP). Module IXSignal Management. London: European Medicines Agency; 2012. Available from: http://www.ema.europa.eu/docs/en_GB/document_ library/Scientific_guideline/2012/06/WC500129138.pdf. Accessed January 17, 2013.

12. Geerse DA, Rutherford P, Bogers JC, Konings CJ. Sterile peritonitis associated with the use of amino-acid solution in eight peritoneal dialysis patients. Perit Dial Int. 2011;31(1):90-91.

13. GlucoseSafety.com [webpage on the Internet]. Extraneal company core data sheet. Deerfield: Baxter Healthcare Corporation; 2007. Available from: http://www.glucosesafety.com/us/pdf/extraneal_pi.pdf. Accessed December 6, 2012.
14. Kroll HR, Maher TR. Significant hypoglycaemia secondary to icodextrin peritoneal dialysate in a diabetic patient. Anesth Analg. 2007;104(6):1473-1474.

15. Basic-Jukic N, Juric I, Racki S, Kes P. Spontaneous tendon ruptures in patients with end-stage renal disease. Kidney Blood Press Res. 2009;32(1):32-36.

16. Garrick R, Kliger A, Stefanchik B. Patient and facility safety in hemodialysis: opportunities and strategies to develop a culture of safety. Clin J Am Soc Nephrol. 2012;7(4):680-688.

\section{Publish your work in this journal}

Drug, Healthcare and Patient Safety is an international, peer-reviewed open-access journal exploring patient safety issues in the healthcare continuum from diagnostic and screening interventions through to treatment, drug therapy and surgery. The journal is characterized by the rapid reporting of reviews, original research, clinical, epidemiological and post-marketing surveillance studies, risk management, health literacy and educational programs across all areas of healthcare delivery. The manuscript management system is completely online and includes a very quick and fair peer-review system. Visit http://www.dovepress.com/ testimonials.php to read real quotes from published authors.

Submit your manuscript here: http://www.dovepress.com/drug-healthcare-and-patient-safety-journal 